\title{
Effet de l'azote et du rayonnement sur le développement des feuilles et de l'épi chez le blé d'hiver : mise en place de l'appareil foliaire et de la structure de l'épi
}

\author{
E Triboï, J Ntonga \\ INRA, station d'agronomie, 12, avenue du Brézet, F 63039 Clermont-Ferrand, France
}

(Reçu le 5 juin 1992; accepté le 20 février 1993)

\begin{abstract}
Résumé - Des plantes monotalles élevées depuis le semis en faible nutrition azotée ou en faible rayonnement ont été transférées en conditions optimales à différents stades du développement. La carence en $\mathrm{N}$ et l'ombrage ont augmenté le thermochrone et leur suppression l'a diminué. L'ombrage a diminué le nombre de feuilles (-2) et la carence en $\mathrm{N}$ le nombre final d'épillets (-4). Le nombre de grains par épi a varié de 54 à 111. L'azote a agi surtout sur la zone basale de l'épi et la lumière sur la moitié supérieure. La suppression de la carence en $\mathrm{N}$ a amélioré la fertilité d'abord à la base de l'épi et ensuite dans la zone médiane et distale. La suppression de l'ombrage a augmenté la fertilité uniformément sur tous les épillets. Après le stade "double ride», la perte de fertilité due à la carence azotée a été proportionnelle à la durée de la carence et n'a pas mis en évidence un stade de développement particulièrement sensible. En revanche, la période allant de la sortie de la dernière feuille à la floraison a présenté une sensibilité supérieure à un complément de lumière. L'existence d'un seuil limite de développement d'une fleur conditionnant sa fertilité est discutée.
\end{abstract}

azote / lumière / thermochrone / structure de l'épi / fertilité de l'épi

Summary - Leaf development and ear structure in wheat as affected by nitrogen and shading. Single mainstems of wheat grown in individual pots and in reduced light intensity (50\%) or without $N$ fertilizer were transferred to optimal conditions (light and nitrogen) at different stages. The effects on thermal time of appearance of successive leaves (thermochron, QT), on number of leaves and spikelets per ear and on grain number, ear structure and fertility of grain sites were analysed.

Nitrogen deficiency $(\mathrm{Nd})$ and shading decreased the rate of leaf appearance (figs 3, 4) and their suppression at terminal spikelet stage raised it (table 1). Ligule appearance was more linearly related to thermal time than lamina appearance, and so was better for the calculation of thermochron value, possibly due to variation in laminal area (fig 10). The total number of primordia (leaves + spikelets) was decreased by both shading (-2) and Nd (-4). Shading decreased the number of leaves (-2) and Nd the number of spikelets (-4) (table II). The number of grains per ear ranged from 51 under fully stressed conditions to 111 under optimal conditions. Continuous Nd acted principally on fertility at the basal part of the ear and shading on the upper part (fig 5). The suppression of Nd increased the fertility successively in basal, median and upper parts of the ear (fig 6) and the suppression of shading increased the fertility uniformly in all spikelets (fig 7).

No stage after double-ridge was significantly more sensitive to nitrogen changes than any other, there being a progressive reduction in grain number with thermal time. In contrast, the period between the appearance of the last leaf and anthesis was more sensitive to light change (fig 8).

The number of sterile florets was similar in all spikelets (2-3) (fig 10) and the hypothesis of a minimum stage of floral development for grain siting has been discussed.

nitrogen / shading / leaf appearance / ear structure / ear fertility 


\section{INTRODUCTION}

La variation du nombre de grains par épi (NGE) est considérée souvent comme un facteur important de variation du nombre de grains par $\mathrm{m}^{2}$ et du rendement à cause du faible pouvoir de compensation du poids du grain (Triboï, 1990).

Hormis le cas d'accidents climatiques provoqués par la sécheresse ou par les températures basses pendant la méiose, la variation du NGE a comme origine la compétition entre plantes ou organes d'une plante pour la lumière et les nutriments ainsi que pour les assimilats (Siddique et al, 1989).

Les différents facteurs du milieu climatique et nutritionnel, en agissant d'une part sur le développement et d'autre part sur le potentiel de croissance, conditionnent l'intensité de la compétition et déterminent le nombre, la taille et le devenir des organes.

Leur effet peut être évalué à partir du thermochrone et de la structure de la plante, représentée par le nombre et la taille des organes végétatifs et reproductifs.

Les travaux concernant la morphogenèse du maître brin et notamment ceux de Vincent et al (1977), Vincent (1982), Malvoisin (1984a, 1984b), Kirby (1984), Delécolle et al (1989), nous offrent un schéma logique d'interprétation des résultats expérimentaux, basé sur les relations existantes entre l'activité de croissance sous-apicale et l'activité organogène des méristèmes terminaux.

Ainsi, la croissance sous-apicale s'effectue avec une régularité remarquable, contrôlée par la température. Elle est indexée au thermochrone (QT), défini comme le temps thermique (base $0^{\circ} \mathrm{C}$ ) nécessaire pour le déroulement d'une étape de croissance : croissance et apparition d'une feuille, création d'une paire de bourgeons floraux (un étage, un pas floral) accompagnée simultanément par l'évolution florale ( 3 ébauches d'anthères) de la paire précédente (Vincent, 1982; Malvoisin, 1984a). Le thermochrone serait fixé précocement et resterait constant (Malvoisin, 1984a; Kirby, 1984). II dépendrait seulement de la vitesse de changement de la longueur du jour au moment de la levée (Kirby et al, 1982; Kirby et al, 1985a); les facteurs nutritionnels tels que l'azote n'auraient d'effet ni sur l'installation du thermochrone, ni sur sa stabilité au long du temps (Miglietta, 1991b). Cependant, Boone et al (1990) montrent un changement bru- tal de $Q T$ sous l'effet de la température et de la concentration en $\mathrm{CO}_{254}$, juste avant l'initiation florale (stade dr). De même, Miglietta (1991a) met en évidence un effet ontogénique qui se manifeste par la diminution progressive du thermochrone de différentes feuilles successives.

Quant au déterminisme du nombre d'organes, notons seulement que, à la suite de l'activité organogène de l'apex, les phytomères produits par la méristème terminal évolueront, selon les conditions du milieu, vers des phytomères végétatifs (ensemble limbe, gaine, nœud et éventuellement entre-nœud) ou floraux (ensemble épillet, nœud de rachis et entre-nœud de rachis).

Les facteurs principaux du milieu qui contrôlent le développement de l'apex et donc la durée et la vitesse d'initiation des phytomères et leur évolution sont l'état de vernalisation de la plante, la température et la photopériode. Leurs effets ont été étudiés par de nombreux auteurs et sont relativement bien connus.

En revanche, les effets des facteurs nutritionnels, substances de croissance incluses, sont nettement moins bien étudiés et souvent contradictoires (Dale et Wilson, 1979). Ils seraient faibles et nettement inférieurs à l'effet cultivar (Frank et Bauer, 1984).

Enfin, au cours de la période florale, a lieu l'organisation de l'épillet. L'initiation et l'évolution des fleurs jusqu'à la fécondation se font à nouveau selon un rythme régulier (Malvoisin, 1984a). Sur les 8-10 fleurs initiées par épillet, seulement 3-6 seront potentiellement fertiles (Kirby, 1984).

Notons aussi que dans un peuplement cultivé en conditions naturelles, l'effet spécifique de chacun des différents facteurs est difficilement séparable, car ils agissent simultanément et en interaction (Puckridge, 1968). De plus, l'expérimentation en plein champ ne permet pas une bonne maîtrise du niveau de facteurs de croissance (Delécolle et Gurnade, 1990) et la présence de plusieurs tiges par plante complique et pourrait même modifier l'effet du facteur expérimenté (Blondon et Morris, 1985, Kirby et al, 1985b).

Ainsi, pour mieux comprendre la compétition dans un peuplement, dans la présente étude nous avons essayé d'apprécier les effets spécifiques de 2 facteurs majeurs du milieu, la lumière et la nutrition azotée, sur le thermochrone, sur le nombre total de phytomères, de feuilles et d'épillets et sur l'élaboration de la structure de l'épi. 


\section{MATÉRIEL ET MÉTHODES}

\section{Dispositif expérimental}

Pour séparer l'effet de la lumière de l'effet de la nutrition azotée, nous avons utilisé une technique expérimentale proche de celle utilisée par Puckridge (1968), en cultivant une plante par pot, mais en multipliant par 3 le volume du sol et en ne gardant que le maitre brin.

Les pots sont des cylindres en polyvinyle de $7,1 \mathrm{~cm}$ de diamètre et de $50 \mathrm{~cm}$ de hauteur, d'un volume d'environ $2000 \mathrm{~cm}^{3}$. Ils sont remplis avec $1080 \mathrm{~g}$ d'un mélange contenant $70 \%$ de terre noire à $45 \%$ d'argile et $30 \%$ de tourbe. Une fertilisation de $250 \mathrm{ppm}$ de $\mathrm{P}_{2} \mathrm{O}_{5}$ et $330 \mathrm{ppm}$ de $\mathrm{K}_{2} \mathrm{O}$ a été apportée à la préparation du mélange.

Le semis a été effectué le 23 décembre 1988 en pots avec des grains prégermés de la variété Camp Rémy, en vue d'assurer une levée régulière. Les plantes ont été élevées jusqu'au stade "b" (ébauche de glumes; un récapitulatif des abréviations est présenté en annexe) en hors-gel sous serre et puis ont été transférées en conditions naturelles. Seul le maître brin a été gardé dans chaque pot, les autres talles et leurs repousses étant supprimées chaque semaine.

Pour éviter les effets de bordure, de l'ombrage réciproque et du microclimat, les pots ont été régulièrement permutés en gardant une équidistance de $30 / 40$ $\mathrm{cm}$ entre 2 pots.

\section{Traitements expérimentaux}

Deux essais ont été conduits simultanément pour étudier l'effet de l'azote et de la lumière selon le stade de développement de la plante.

Pour cela, des plantes élevées depuis le semis en faible nutrition azotée (essai 1) ou en faible rayonnement incident (essai 2) ont été mises en conditions optimales de nutrition azotée et en lumière naturelle à partir de différents stades de développement, conformément aux schémas présentés dans la figure $1 \mathrm{a}, \mathrm{b}$. Ainsi l'effet de l'azote ou de la lumière apporté(e) à partir d'un certain stade de développement peut être comparé aux 3 témoins, cultivés depuis le semis en :

- lumière naturelle et azote optimal : LN,

- lumière naturelle et faible nutrition azotée : Ln,

- azote optimal et lumière réduite : NI

La réduction de la lumière a été réalisée par l'installation d'une toile de polypropylène noire à $1 \mathrm{~m}$ audessus du sommet des plantes. Le dispositif a permis de réduire de $60 \%$ le rayonnement incident, sans modifier le régime thermique (fig 2).

L'alimentation minérale azotée dite optimale a consisté en l'apport de $25 \mathrm{mg}$ de $\mathrm{N}$ par pot sous forme de nitrate d'ammonium dilué dans $100 \mathrm{~cm}^{3}$ d'eau. Ces apports sont effectués à l'apparition de chaque feuille à
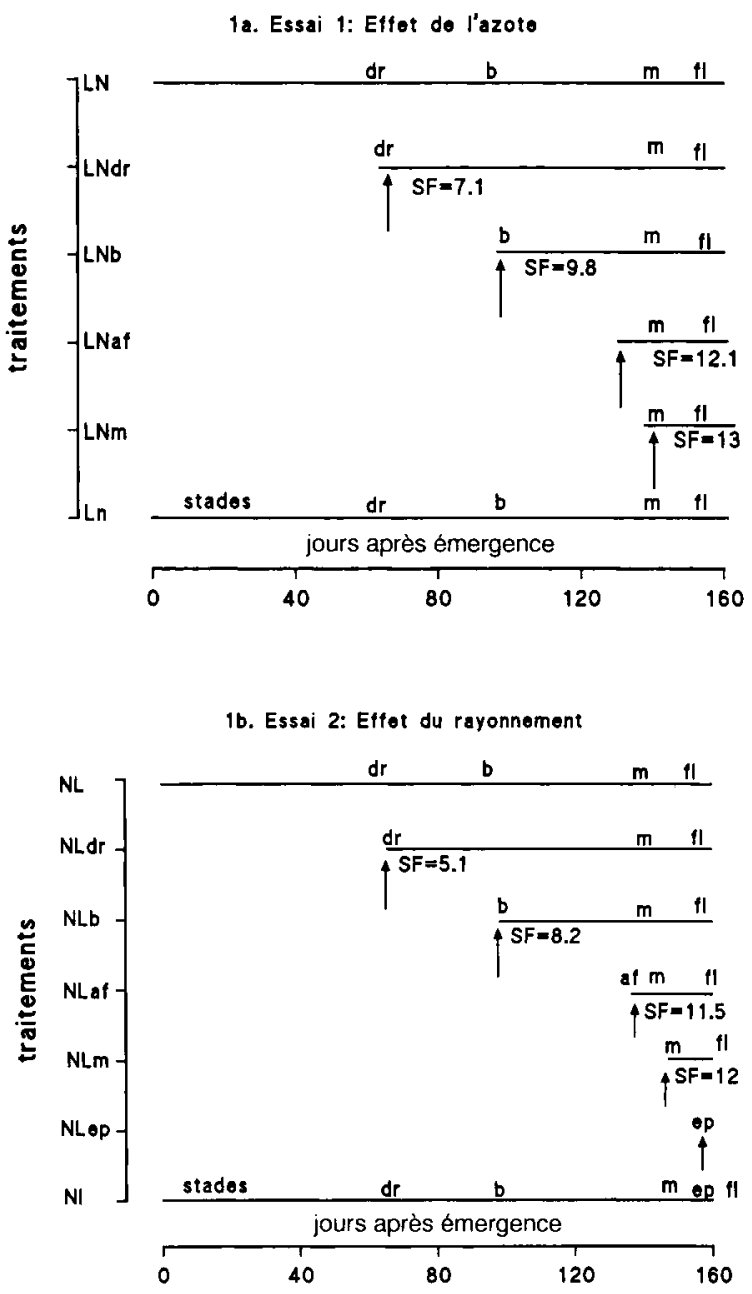

Fig 1. Schéma expérimental : $\mathbf{a}=$ effet de l'azote, $\mathbf{b}=$ effet du rayonnement; I : dates de transfert en nutrition $\mathrm{N}$ optimale (essai 1a) ou en lumière naturelle (essai 1b); les abréviations des stades de développement (dr, b, af, $m$, ep, fl) et des traitements (NL, Ln, NI, NLi ou LNi) sont explicitées dans l'annexe.

partir de la $3^{e}$ jusqu'à la $7^{e}$, puis toutes les semaines jusqu'au 26 juin 1989. Les pots non fertilisés reçoivent uniquement les $100 \mathrm{~cm}^{3}$ d'eau.

\section{Échantillonnage et observations}

Le développement a été apprécié par voie non destructrice au niveau foliaire, et destructrice au niveau de l'épi.

L'évolution du stade foliaire a été suivie sur un échantillon de 10 plantes sur lesquelles on a marqué la base des limbes 3, 6 et 9, dans les 3 témoins (LN, $\mathrm{Ln}, \mathrm{NI}$ ) et dans les traitements transférés au stade "b" en conditions optimales. Le stade foliaire a été apprécié par 2 méthodes en notant soit l'émission du limbe soit l'émission de la gaine ou de la ligule (Haun, 1973; Vincent et al, 1977). 


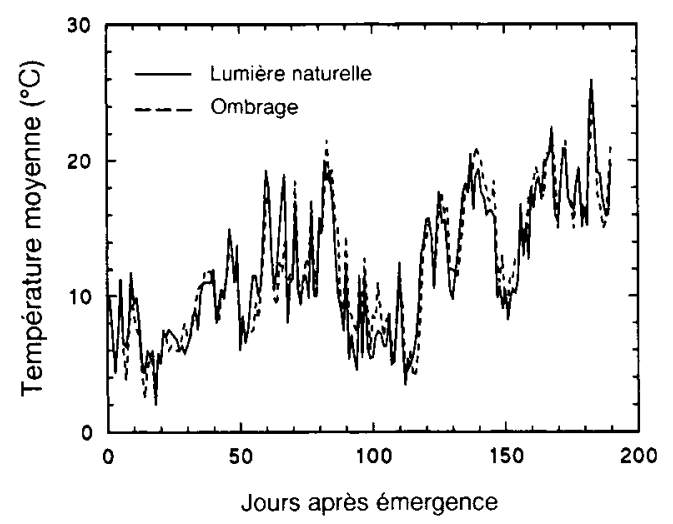

Fig 2. Évolution comparée de la température de l'air sous ombrage et sous lumière naturelle.

Le thermochrone a été calculé par régression linéaire du stade foliaire en fonction du temps thermique exprimé en ${ }^{\circ} \mathrm{C}$.j en base $0^{\circ} \mathrm{C}$ (fig 3 et 4 ).

Parallèlement, on a suivi le développement de l'épi sur 1 à 2 plantes pour déterminer le moment de transfert et sur 5 plantes pour déterminer le stade au moment du transfert. Les stades notés ont été : doublesrides $(d r)$, ébauche de glumes $(b)$, méiose $(m)$ notée quand la ligule de la dernière feuille est dégagée de quelques cm (Malvoisin, 1984b), épiaison (ep), et la floraison (fl) correspondant à la sortie des étamines (figs 1 et 2). Sur ces échantillons on a mesuré les surfaces des limbes par planimétrie optique.

À la récolte, sur au moins 5 plantes par traitement, on a analysé la structure de l'épi en notant le nombre final d'épillets, le nombre de fleurs, de grains et leur position dans chaque épillet.

\section{RÉSULTATS}

\section{Effet de la lumière et de l'azote sur le développement : déterminisme du nombre total de phytomères, de feuilles et d'épillets par épi}

\section{Effet sur le thermochrone (QT)}

Notons tout d'abord une relation bilinéaire entre le stade foliaire déterminé à partir de l'émergence du limbe et le temps thermique : les premières 5-6 feuilles apparaissent plus rapidement que les feuilles suivantes (fig 3 ). Le thermochrone des premières feuilles est donc plus faible que celui des feuilles suivantes. En revanche, si le critère choisi est l'apparition de la ligule, cette relation est linéaire, au moins à par-

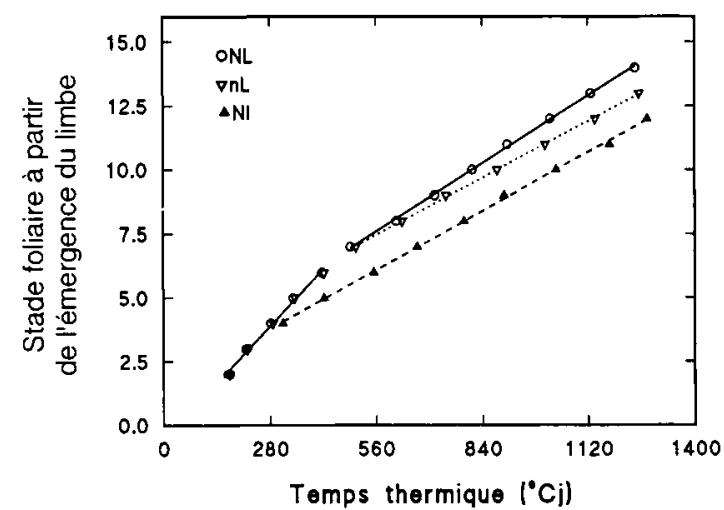

Fig 3. Effet de l'azote et de la lumière sur l'évolution du stade foliaire déterminé à partir de l'émergence du limbe foliaire. NL, Ln, NI : abréviations explicitées dans l'annexe.

tir de l'émergence de la gaine de la feuille 4 (fig 4).

En ce qui concerne l'effet des traitements, signalons une augmentation du thermochrone sous l'effet de la carence azotée et de l'ombrage, car la pente de la droite de régression diminue dans l'ordre NL, NI et Ln (figs 3 et 4).

Le tableau I présente une vision synthétique des valeurs des thermochrones calculés pour l'ensemble des situations examinées. II permet de mettre en évidence :

- l'effet d'une carence (azote ou lumière) continuelle depuis le semis. Par rapport à l'optimum $L N$, le $Q T$ de $L n$ et $N$ l augmente. L'effet lumière semble plus important que l'effet azote, quelle que soit la méthode de calcul;

- l'effet de l'apport d'azote ou de lumière à partir du stade b. Le $Q T$ pour les traitements NLb et

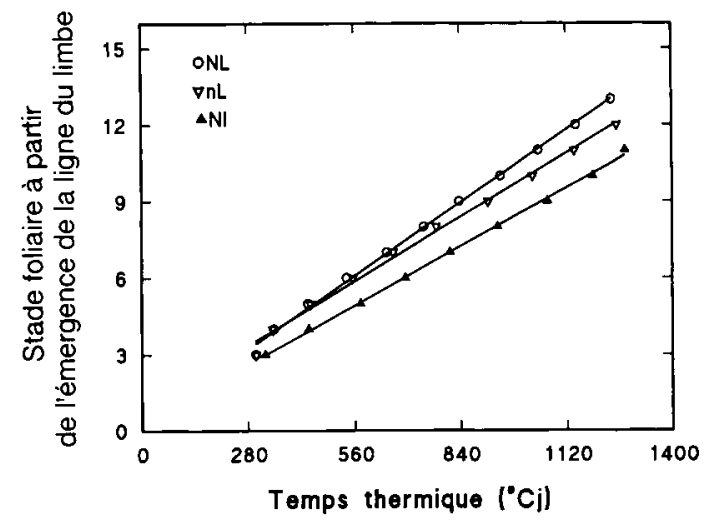

Fig 4. Effet de l'azote et de la lumière sur l'évolution du stade foliaire déterminé à partir de l'émission de la ligule du limbe foliaire (gaine). $\mathrm{NL}, \mathrm{Ln}, \mathrm{NI}=$ abréviations explicitées dans l'annexe. 
Tableau I. Effet de l'azote et du rayonnement sur le thermochrone.

\begin{tabular}{|c|c|c|c|c|c|c|c|c|}
\hline & \multirow[t]{3}{*}{ NF } & \multirow[t]{3}{*}{ NO } & \multirow[t]{3}{*}{$n$} & \multicolumn{4}{|c|}{ Thermochrone (QT) calculé à partir de l'émergence } & \multirow{3}{*}{$\begin{array}{c}\text { Comparaison } \\
\text { QTL/QTG } \\
S(P<0,05)\end{array}$} \\
\hline & & & & \multicolumn{2}{|c|}{ du limbe } & \multicolumn{2}{|c|}{ de la gaine } & \\
\hline & & & & QTL & SE $T H$ & $\begin{array}{c}\text { QTG } \\
(\mathrm{P}>0,05)\end{array}$ & SE TH & \\
\hline \multicolumn{9}{|c|}{ Effet de la nutrition azotée } \\
\hline LN & 14 & 13 & 6 & 91,5 & $0,6921^{a}$ & 100,1 & $0,7720 \mathrm{a}$ & S \\
\hline LN4 & 14 & 4 & 6 & 111,7 & $2,7645^{b}$ & 97,6 & $2,7171 \mathrm{a}$ & $\mathrm{S}$ \\
\hline Ln & 13 & 12 & 9 & 101,1 & $0,7123^{c}$ & 115,6 & $0,8683^{b}$ & $S$ \\
\hline $\operatorname{Ln} 4$ & 13 & 14 & 9 & 126,3 & $2,9177^{d}$ & 114,5 & $2,2013^{b}$ & S \\
\hline $\mathrm{LNb}$ & 13 & 4 & 3 & 114,9 & $3,4049^{b}$ & 100,9 & $8,1561 \mathrm{a}$ & NS \\
\hline \multicolumn{9}{|c|}{ Effet du rayonnement } \\
\hline NL & 14 & 13 & 6 & 91,5 & $0,6921 \mathrm{a}$ & 100,1 & $0,7720^{a}$ & $S$ \\
\hline NL4 & 14 & 4 & 6 & 111,7 & $2,7645^{b}$ & 97,6 & $2,7171 \mathrm{a}$ & $S$ \\
\hline $\mathrm{Nl}$ & 12 & 11 & 8 & 115,5 & $1,1610 \mathrm{~b}$ & 120,6 & $1,1004^{b}$ & $S$ \\
\hline $\mathrm{N} / 4$ & 12 & 4 & 8 & 125,8 & $3,0269 \mathrm{c}$ & 113,2 & $3,4921 \mathrm{~b}$ & NS \\
\hline NLb & 12 & 4 & 3 & 108,2 & $6,8609 \mathrm{~b}$ & 89,2 & $10,700^{a}$ & NS \\
\hline
\end{tabular}

Nombre de feuilles émises : total (NF) ou pendant la période de calcul de $Q T(N O)$; $T H=$ test d'homogénéité : les traitements suivis de la même lettre en sont pas différents; $n=$ effectif des plantes utilisé dans le calcul du stade foliaire. $S E=$ écart type.

LNb a été calculé à partir de l'émission des 4 dernières feuilles. Les $Q T$ des traitements NLb et LNb sont égaux au QT du NL (NL4) et plus faibles que le $Q T$ des traitements carencés en $\mathrm{N}$ ou en L depuis le semis (Ln4 et NI4);

- le critère "émission de la gaine ou de la ligule" est mieux adapté pour calculer $Q T$ que «l'émission du limbe», car il présente une meilleure linéarité avec le temps thermique. Chez les 3 témoins, les $Q T$ calculés à partir de l'émission des 4 dernières feuilles (NL4, Ln4 et NI4) sont supérieurs aux $Q T$ globaux (NL, Ln et NI) pour le critère "sortie du limbe» et égaux pour le critère "sortie de la ligule».

\section{Effet sur le nombre total de phytomères, de feuilles et d'épillets par épi (tableau II)}

La production des phytomères a lieu pendant une période allant jusqu'à la différenciation de l'épillet terminal et elle est indexée au temps thermique (Vincent, 1982; Malvoisin, 1984a; Delécolle et al, 1989; Kirby et al, 1989). Le nombre total produit correspond à la somme du nombre d'organes auxquels ils donnent naissance, donc à la somme du nombre de feuilles et d'épillets.

Dans notre expérimentation, 41 phytomères ont été enregistrés dans le traitement non limitant
Tableau II. Effet de la lumière et de l'azote sur la structure de l'épi.

\begin{tabular}{|c|c|c|}
\hline $\begin{array}{rc}\text { Traitement } N b & N b \\
\text { feuilles épillets/épi } \\
\text { Total Stériles }\end{array}$ & $\begin{array}{c}\mathrm{Nb} \\
\text { phytomères }\end{array}$ & $\begin{array}{l}\mathrm{Nb} \\
\text { grains/épi } \\
T H\end{array}$ \\
\hline
\end{tabular}

\begin{tabular}{|c|c|c|c|c|c|}
\hline \multicolumn{6}{|c|}{ Effet lumière } \\
\hline $\mathrm{NL}$ & 14 & 26,8 & 0 & 41 & $110,8 \mathrm{a}$ \\
\hline NLdr & 12 & 27,0 & 0 & 39 & $97,7 a, b$ \\
\hline $\mathrm{NLb}$ & 12 & 26.5 & 1 & 39 & $85,5^{b, c}$ \\
\hline NLaf & 12 & 27,0 & 0 & 39 & 86,7 b,c \\
\hline NLm & 12 & 26,7 & 3 & 39 & $76,6 \mathrm{c}, \mathrm{d}$ \\
\hline NLep & 12 & 26,6 & 6 & 39 & $60,4 d, e$ \\
\hline NLfl & 12 & 26,7 & 6 & 39 & $55,0^{e}$ \\
\hline $\mathrm{NI}$ & 12 & 26,5 & 6 & 39 & 54,1 e \\
\hline \multicolumn{6}{|c|}{ Effet azote } \\
\hline LN & 14 & 26,8 & 0 & 41 & $110,8 \mathrm{a}$ \\
\hline LNdr & 14 & 26,7 & 0 & 41 & 112,0 a \\
\hline $\mathrm{LNb}$ & 13 & 23,8 & 0 & 37 & $87,1^{b}$ \\
\hline LNaf & 13 & 23,8 & 0 & 37 & $79,8^{b, c}$ \\
\hline LNm & 13 & 24,2 & 0 & 37 & $67,4^{c, d}$ \\
\hline LNfl & 13 & 23,4 & 1 & 36 & $56,2 \mathrm{~d}, \mathrm{e}$ \\
\hline $\operatorname{Ln}$ & 13 & 23,9 & 2 & 37 & $50,8^{e}$ \\
\hline
\end{tabular}

NL. Ils se sont transformés en 14 feuilles et 27 épillets.

L'ombrage (NI) a diminué de 2 le nombre total de phytomères (39). Cela s'est répercuté sur le 
nombre de feuilles; le nombre d'épillets est resté constant (27). L'apport d'un supplément d'éclairage à différents stades n'a pas modifié ces valeurs.

En revanche, l'azote a eu un effet plus prononcé car il a diminué de 4 le nombre total de phytomères; cela s'est répercuté plus sur la production d'épillets $(-3$ à -4$)$ que sur le nombre de feuilles $(-1)$. Contrairement à l'effet lumière, un apport d'azote précoce au stade double ride $\left(547^{\circ} \mathrm{C}\right.$.j ou stade foliaire 7,1 ) est encore efficace tant en ce qui concerne la production de feuilles que d'épillets, leur nombre étant identique au traitement LN. Les apports plus tardifs n'ont plus d'effet, le nombre de phytomères, de feuilles et d'épillets étant égal à celui du traitement Ln.

\section{Effet de la lumière et de l'azote sur la fertilité de l'épi : déterminisme du nombre de grains par épillet et par épi}

\section{Effet d'une carence imposée depuis la levée}

Le traitement non limitant NL a produit 111 grains/épi (NGE) (tableau II). Tous les épillets de l'épi ont été fertiles. Cependant, l'analyse de l'épi nous révèle 3 zones de fertilité (fig 5) :

- la base de l'épi, les premiers 2 épillets ont en moyenne 3,5 à 4,0 grains par épillet;

- la zone médiane de l'épi comprenant les épillets 3 à 17 qui possèdent 4,5 à 5,0 grains par épillet;

- la zone distale ou le sommet, dont la fertilité diminue d'environ 0,2 grain par épillet pour arriver à 2 grains dans l'épillet 26 . L'épillet terminal 27 ne possède qu'un seul grain.

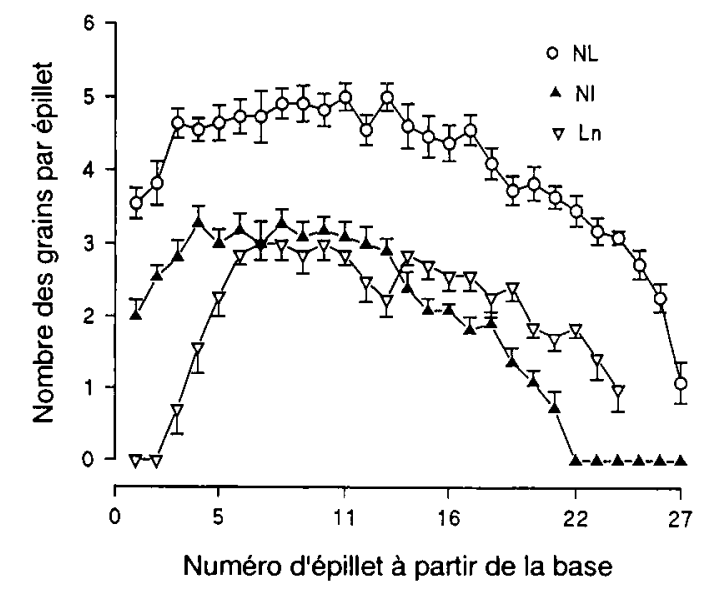

Fig 5. Effet de l'azote et de la lumière sur la fertilité des épillets. NL, Ln, NI : abréviations explicitées dans l'annexe.
La carence en azote ou en lumière depuis le semis a diminué la fertilité de l'épi de plus de la moitié : 50,8 grains pour $\mathrm{Ln}$ et 54,1 pour $\mathrm{NI}$ (tableau II).

Cette diminution spectaculaire n'est pas uniformément répartie sur tous les épillets de l'épi. Les 3 zones sont maintenues mais l'effet sur NGE diffère avec le facteur.

Ainsi, la carence azotée (Ln) produit une très forte baisse de la fertilité à la base de l'épi : les premiers 2 épillets sont stériles et les suivants ont une fertilité qui augmente progressivement pour atteindre 3 grains au $6^{e}$ épillet. La zone médiane, la plus fertile, est plus étroite et elle possède un maximum de 3 grains par épillet. Enfin, dans la zone distale, la fertilité baisse mais avec une intensité très voisine du traitement NL.

En revanche l'effet de l'ombrage est nettement plus prononcé dans la zone distale que basale. Les derniers 6 épillets sont stériles et la perte de fertilité dans la zone $13-22$ est plus prononcée que dans $\mathrm{NL}$ et $\mathrm{Ln}$. La zone basale a un comportement proche de celle de NL car elle ne concerne que les 3 premiers épillets et elle est très fertile, contenant 2-3 grains/épillet. La zone médiane présente presque la même fertilité, environ 3 grains/épillet. Elle comporte le même nombre d'épillets, environ 10, mais décalé vers le sommet de l'épi dans $L n$ et vers la base dans NI.

II résulte donc qu'une carence azotée a un effet négatif plus prononcé sur la zone basale de l'épi tandis que l'ombrage agit essentiellement sur la moitié supérieure (fig 5).

\section{Effet de la date de suppression d'une carence azotée ou de l'ombrage sur la structure de l'épi. Les périodes sensibles}

\section{La structure de l'épi}

L'azote, apporté tardivement, à la floraison, n'a plus d'effet sur la fertilité de l'épi. Apporté à la méiose, il améliore la fertilité des 10 premiers épillets et notamment de ceux de la base de l'épi (fig 6); le nombre de grains par épi augmente de 17 grains (tableau II). Lorsqu'on apporte $\mathrm{N}$ un thermochrone plus tôt, à la sortie de l'avantdernière feuille, on récupère encore 12 grains, principalement par l'amélioration de la fertilité dans la zone médiane. Avec des apports plus précoces, aux stades $b$ et $d r$, on gagne encore respectivement 7 et 32 grains par épi, en améliorant la fertilité de tout l'épi et notamment de la 


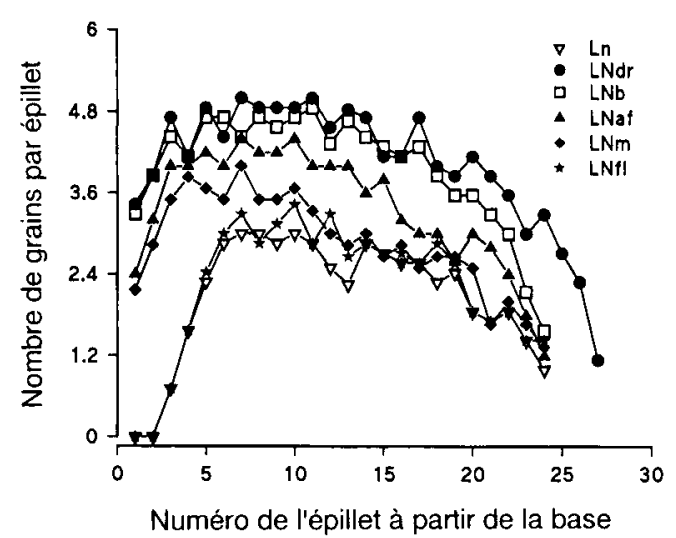

Fig 6. Effet de la date de suppression de la carence azotée sur la fertilité des épillets. Les abréviations Ln et LNi sont explicitées dans l'annexe.

moitié supérieure. La carence dans la période levée - dr n'a pas eu d'effet sur la fertilité de l'épi, car le traitement $L N d r$ a produit le même $N G E$ que le témoin $\mathrm{LN}$.

II apparaît donc que l'azote agit d'abord sur les épillets de la base de l'épi et ensuite sur la zone médiane et supérieure.

Quant à l'effet de la lumière, ce mode d'action préférentiel n'est plus enregistré, car l'éclairement à différents stades, méiose, b, dr, augmente la fertilité de l'épi uniformément sur tous les épillets (fig 7).

\section{Les stades sensibles}

La suppression de la carence azotée ou de l'ombrage à différents stades du développement modifie la fertilité de l'épi. À partir du nombre moyen de grains par épi (tableau II), on peut évaluer l'effet de la carence en calculant un taux de perte

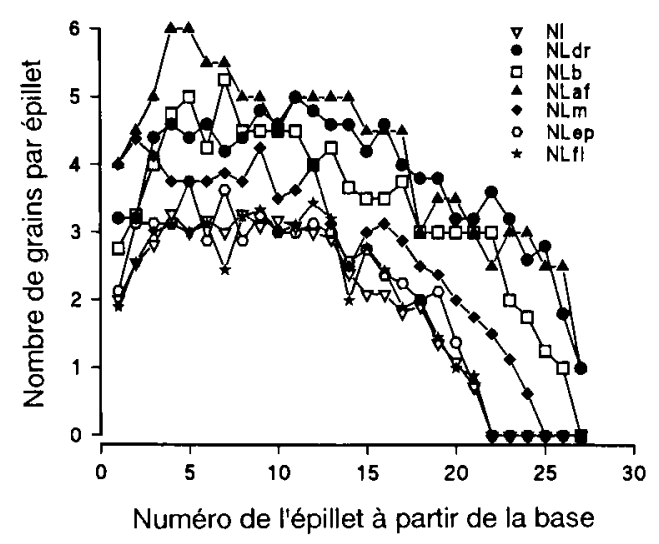

Fig 7. Effet de la date de suppression de l'ombrage sur la structure de l'épi. Les abréviations $\mathrm{NI}$ et $\mathrm{NLi}$ sont explicitées dans l'annexe.
( $T P$ avec $T P l$ pour la lumière et $T P n$ pour l'azote) et l'effet de la suppression, en calculant un taux de récupération ( $T R$ avec $T R /$ pour la lumière et $T R n$ pour l'azote) :

$$
\begin{gathered}
T P n=(L N-L N I) /(L N-L n) \\
\quad \text { et } T P I=(L N-N L I) /(L N-N) \\
T R n=(L N i-L n) /(L N-L n) \\
\quad \text { et } T R I=(N L i-N I) /(L N-N)
\end{gathered}
$$

Ces données, présentées graphiquement (fig 8), mettent en évidence une grande similitude d'action des 2 facteurs testés.

L'effet négatif d'une carence en $\mathrm{N}$ se manifeste essentiellement à partir du stade dr. Ensuite les pertes de fertilité varient remarquablement linéairement avec la durée thermique. Dans cet intervalle, il n'y a donc pas un stade de développement particulièrement sensible à la carence azotée. Rawson et Bagga (1979), en étudiant l'effet thermique, ont enregistré un résultat similaire : absence d'un stade particulièrement sensible, car la diminution de la fertilité de l'épi a été proportionnelle au temps de séjour en température élevée.

Quant à l'effet lumière, notons d'abord une dispersion plus importante des données. L'effet négatif d'une carence dans la période levée $-d r$ doit être confirmé car la perte de 13,1 grains, ce qui représente $23,1 \%$ de la variation maximale du nombre de grains, n'est pas significativement différente de NL.

Soulignons aussi l'efficacité supérieure d'un apport supplémentaire d'éclairement pendant la période sortie du limbe de la dernière feuille méiose - floraison et la relative insensibilité à

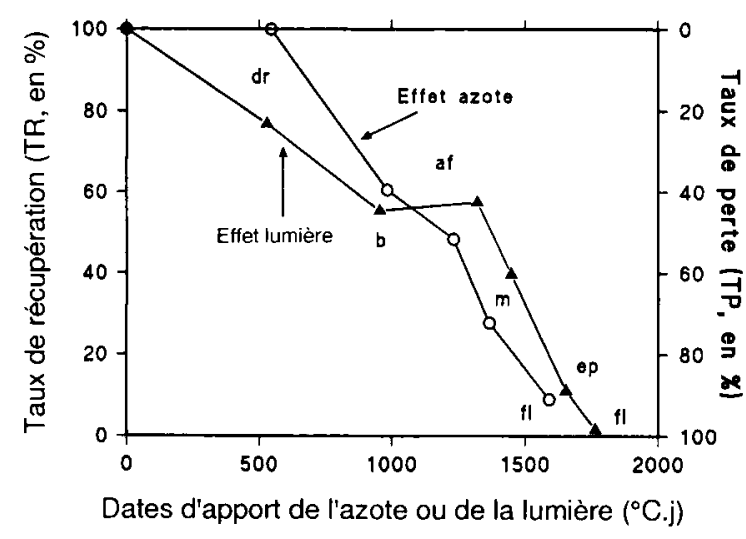

Fig 8. Effet relatif de la suppression d'une carence azotée et de l'ombrage. $d r, b, a f, m, e p, f l=$ stades de développement, conforme à l'annexe; TP et TR = définis dans le texte. 
cet apport de la période apparition des ébauches de glumes - sortie du limbe de la dernière feuille. Ces données sont similaires à celles obtenues en essais au champ par Fischer (1985).

\section{DISCUSSION ET CONCLUSION}

\section{L'effet sur le thermochrone}

La plupart des auteurs qui ont étudié le thermochrone $(Q T)$ considèrent qu'il est déterminé précocement, qu'il reste stable tout au long du parcours (Gallagher, 1979; Delécolle et Gurnade, 1980) et qu'il est insensible aux variations qui affectent l'activité organogène apicale (Malvoisin, 1984a).

Or, nos données mettent en cause la stabilité de $Q T$, tant entre différentes situations (effet de l'azote et de l'ombrage) que tout au long du parcours.

La variation de $Q T$ sous l'effet du milieu, par exemple la date de semis, a déjà été signalée (Kirby et al, 1982 et 1985a). En revanche, on a considéré que les facteurs nutritionnels, tels que l'azote, n'ont pas d'effet sur le rythme d'apparition des feuilles (Willington et Biscoe, 1983 cités par Kirby et al, 1985a; Delécolle et Gurnade, 1980).

Quant à la stabilité de $Q T$ tout au long du parcours, elle est mise en cause aussi par d'autres auteurs.

Ainsi, chez Kirby et al (1985a), la relation entre le nombre de feuilles et le temps thermique, malgré le fait qu'elle était hautement significative, présentait une déviation systématique de la linéarité; le changement de la température de base de 0 à $5{ }^{\circ} \mathrm{C}$ a éliminé cette déviation, sans cependant apporter de contribution à la compréhension du phénomène. Un changement brutal de $Q T$ sous l'effet de la température et de la concentration en $\mathrm{CO}_{2}$ a été enregistré aussi par Boone et al (1990), juste avant le stade dr.

Hormis les effets directs de ces facteurs, ces variations pourraient s'expliquer, au moins en partie, par les mécanismes de croissance de la feuille.

Ainsi, le temps nécessaire pour l'apparition d'une feuille dépend d'une part de la distance à parcourir par le limbe en expansion active et d'autre part de la vitesse d'expansion du limbe; par exemple, la distance à parcourir par le limbe en expansion rapide de la feuille «i» est repré- sentée par la longueur de la gaine enveloppante « $-1 »$, qui a fini son expansion. Celle-ci augmente exponentiellement avec l'apparition des feuilles successives et elle est influencée par le milieu (Hay, 1986). Un QT constant nécessite donc des relations allométriques non variables entre la croissance des différentes feuilles de la plante, fait peu probable à cause de l'ontogénie de la plante et de l'effet du milieu sur la taille des organes.

En effet, Gallagher (1979) met en évidence une augmentation de la longueur du limbe essentiellement à partir de la feuille 6 jusqu'à l'avant-dernière.

Les mesures de surfaces foliaires que nous avons effectuées dans notre essai confirment et précisent le rôle du milieu dans le déterminisme de la taille de l'organe (fig 9) :

- les surfaces augmentent à partir du limbe 5 ou 6 jusqu'à l'avant-dernier,

- la carence azotée depuis le semis diminue la surface des limbes et l'ombrage l'augmente. L'effet de l'ombrage se manifeste plus précocement que celui de l'azote. Ces résultats sont en accord avec ceux obtenus par Puckridge (1968),

- la suppression d'une carence azotée ou de l'ombrage modifie la surface des limbes non émis au moment du traitement. L'effet le plus marquant a lieu sur le $3^{e}$ limbe sorti après l'apport d'azote ou de lumière. Au moment du traitement, ce limbe se trouvait à environ $2 Q T$ avant son émergence, donc en phase de multiplication cellulaire ou début de l'expansion active.

Ces effets pourraient très bien être à l'origine de la variation de $Q T$, car dans son calcul on fait appel à des caractéristiques des organes déter-

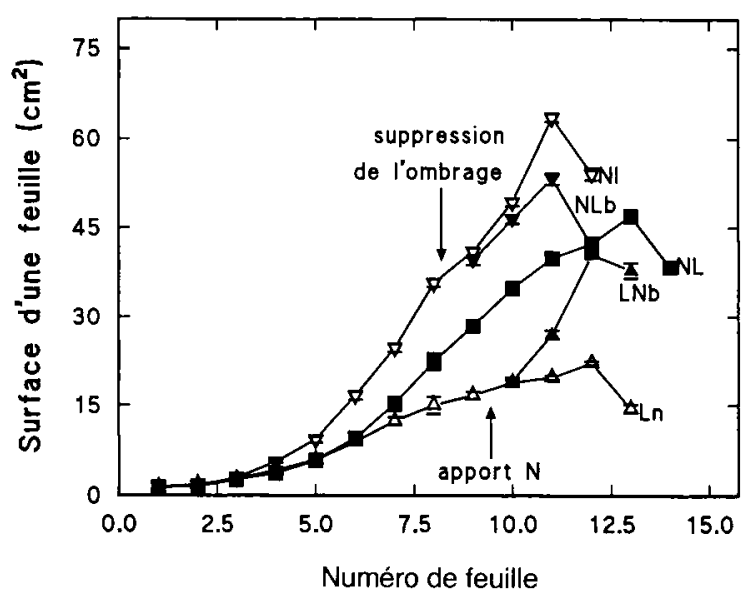

Fig 9. Effet de l'azote et de l'ombrage sur la surface foliaire des feuilles. NL, Ln, NI, NLb, LNb = abréviations explicitées dans l'annexe. 
minées à différents instants «t», donc sous des conditions environnementales variables, qui de plus, se superposent à l'effet ontogénique. Par exemple, la longueur des gaines émises avant la montaison, ou la distance à parcourir pour l'émission des premiers 5-6 limbes reste relativement constante malgré une augmentation des surfaces et donc les longueurs du limbe; il résulterait donc une émergence plus rapide de la feuille ou un QT plus faible.

En revanche, si on utilise comme critère d'observation l'émergence de la gaine, cet effet serait atténué car le rapport allométrique des gaines pourrait être plus stable que celui limbe/gaine.

Ces hypothèses pourraient ainsi expliquer l'augmentation de $Q T$ sous l'effet d'une carence azotée ou de l'ombrage : le rapport entre la longueur de la gaine « $j-1$ » et la vitesse d'expansion du limbe «i» augmente, ou, encore, l'augmentation (effet ontogénique, ombrage) ou la diminution (carence azotée) de la vitesse d'expansion du limbe ne compense pas la variation de la distance à parcourir.

\section{Le déterminisme du nombre de feuilles et d'épillets par épi}

Le nombre d'organes, feuilles et épillets, dépend d'une part du nombre total de phytomères initiés, et d'autre part de leur évolution.

D'après Vincent (1982) et Malvoisin (1984a), les phytomères initiés pendant la période végétative allant jusqu'à l'apparition des premières ébauches d'entre-nœuds (stade A1 ou At), évolueront en phytomères végétatifs ( 3 primordia sont déjà présents dans l'embryon) et donneront naissance à un ensemble limbe, gaine, nœud, et entre-nœud. Le rythme d'initiation est lent, environ 2 phytomères par QT. II est indépendant de la longueur du jour, mais augmente avec la température (Miglietta, 1989).

Après le stade Al ou At, l'initiation des phytomères s'accélère environ par 3 (Dale et Wilson, 1979; Vincent, 1982). Ce rapport dépend de la date de semis et de la variété (Delécolle et al, 1989). L'initiation s'arrête à la transformation de l'apex terminal en épillet terminal (ET). Les initiums formés dans cette période d'initiation de l'épi ou de transition évolueront ultérieurement en épillets associés à un article de rachis.

Pendant cette période, après l'initiation d'environ $3 / 4$ du nombre total de phytomères, commence la différenciation des épillets vers le mi- lieu de l'ensemble des phytomères indifférenciés par la dominance apicale. Ce stade, correspondant à l'initiation florale (Kirby, 1974; Blondon et Morris, 1985), noté Ae, A2 ou dr (double ride), malgré le fait qu'il n'a pas une réelle signification dans l'évolution végétative ou reproductrice du phytomère, est utilisé souvent comme repère dans l'analyse de la morphogenèse du blé car il est facilement identifiable (Delécolle et al, 1989). Aussi, certains auteurs préfèrent-ils utiliser le stade "commencement de l'initiation de l'épi» ou le moment de l'initiation du premier épillet. Cependant, sa date d'apparition ne peut être déterminée que rétrospectivement, après que le nombre total des feuilles soit connu (Kirby, 1984; Delécolle et al, 1989).

Notons aussi que le devenir des derniers phytomères initiés pendant la période végétative est incertain, car, selon l'environnement, ils peuvent évoluer vers un organe végétatif ou reproductif (Griffiths et al, 1985; Delécolle et al, 1989).

D'une manière générale, les températures vernalisantes suivies par des jours longs favorisent l'induction florale (Griffiths et al, 1985; Porter et al, 1987). Une déficience en vernalisation et/ou les jours courts produisent une extension considérable de la transition, malgré le retard de son déclenchement, ce qui conduit à l'augmentation du nombre de feuilles, car l'initiation florale est nettement plus retardée. La durée du stade $\mathrm{dr}$ à l'épiaison reste relativement constante (Griffiths et al, 1985). Cependant, l'action du froid peut être remplacée par un fort éclairement dont la valeur des radiations photosynthétiquement actives semble seule importante (Blondon et Morris, 1985), ou, dans certaines conditions, les jours courts peuvent remplacer l'effet des températures basses en provoquant l'initiation florale (Evans, 1987). En photopériode continue, le développement est plus rapide et le nombre d'épillets par épi diminue (Langer et Hanif, 1973; Rahman et Wilson, 1978; Dale et Wilson, 1979). Une augmentation des températures sous une photopériode normale (12,5-14,5 h) ne modifie pas le nombre de phytomères présents au stade "initiation florale", mais change leur évolution en faveur des feuilles (Rahman et Wilson, 1978). Après l'initiation florale, les variables les plus significatives intervenant dans le déterminisme du nombre d'épillets sont le nombre d'épillets initiés par jour (vitesse d'initiation) et la durée d'initiation. Ils sont souvent en interaction négative, ce qui confère au nombre d'épillets une certaine stabilité (Rahman et Wilson, 1978; Kirby et al, 1989). 
Hormis ces facteurs qui contrôlent le développement, la transformation d'un primordium en feuille ou épillet peut dépendre aussi des facteurs nutritionnels.

Par exemple, Puckridge (1968) mentionne un rôle positif de l'azote sur le nombre d'épillets (+4) et pratiquement nul sur le nombre de feuilles. L'augmentation de la population d'épis, ce qui correspond à une diminution du rayonnement disponible par tige, n'a modifié ni le nombre de feuilles ni d'épillets.

En revanche Holmes (1973) enregistre chez 2 génotypes (Marquis et Pitic) un effet positif important de l'azote tant sur la durée que sur la production des primordia, qui se traduit par une augmentation du nombre de feuilles par plante $(+1$ et +3$)$ et des épillets par épi $(+5$ et +17$)$. Par contre, chez l'orge carencée en azote, Dale et Wilson (1978) enregistrent une diminution du nombre de feuilles par la modification du développement vers les structures reproductrices. Enfin, Kemp et Whingwiri (1980), cités par McMaster et al (1987), observent une diminution du nombre d'épillets chez les plantes ombragées après le stade dr.

Nos données, obtenues en conditions expérimentales visant à éliminer l'effet "tallage et peuplement", confirment certains de ces résultats. Elles montrent un effet assez marquant des 2 facteurs, azote et lumière, sur le développement foliaire et de l'épi.

Notons d'abord que la carence en azote et l'ombrage depuis le semis ont diminué le nombre total des primordia initiés et leur répartition entre feuilles et épillets. L'ombrage a diminué le nombre de primordiums de 2; cela s'est répercuté uniquement sur le nombre de feuilles. En revanche, la carence azotée a diminué le nombre total des primordia de 4 , dont 1 feuille et 3 épillets.

II semble donc que les 2 facteurs n'agissent pas de même manière, l'effet de l'ombrage étant plus précoce que celui de l'azote. Cela est en concordance avec les résultats de Puckridge (1968) et Dale et Wilson (1978), qui ont constaté un effet $\mathrm{N}$ seulement à partir de la feuille 3 , car la quantité d'azote du grain serait suffisante pour couvrir les besoins pour la croissance des 3 premières feuilles. En considérant une production de 2 phytomères par thermochrone, il résulte que cette première partie de la période juvénile correspond à l'initiation d'au moins 6 phytomères (Vincent, 1982; Malvoisin, 1984a) ou de 9 feuilles potentielles, car 3 étaient déjà présentes dans l'embryon. Dans la période de transition, la carence en $\mathrm{N}$ s'accentue, car d'une part les besoins deviennent plus importants et d'autre part les ressources s'épuisent. Ainsi, l'élaboration du nombre d'épillets se place dans une période plus critique que celle correspondant à l'élaboration du nombre de feuilles, d'où un effet plus important sur le nombre d'épillets.

En conditions d'ombrage, la plante s'adapte aux ressources disponibles en augmentant la surface foliaire et, ainsi, l'effet négatif pendant la période de transition pourrait être plus faible.

\section{La période florale: déterminisme du nombre de fleur et des grains par épillet}

Concernant cette période plusieurs faits majeurs sont à noter :

- l'épi dans cette période possède une très forte capacité de réaction aux changements nutritionnels, car le nombre de grains a varié de 51 , en carence en $\mathrm{N}$ depuis le semis, à 111 dans le traitement non limitant;

- à cause de la compétition entre les épillets ou entre les fleurs d'un épillet, la fertilité des épillets n'est pas homogène tout le long de l'épi;

- l'effet de cette compétition sur la fertilité des épillets au long de l'épi diffère avec le facteur étudié; la carence azotée a un effet négatif plus prononcé sur la zone basale de l'épi tandis que l'ombrage agit plus sur la moitié supérieure. Quoique moins net, ce type de réponse a été déjà signalé par Kemp et Whingwiri (1980) cité par McMaster et al (1987) pour l'ombrage et Puckridge (1968) et Langer et Hanif (1973) pour l'azote.

$\grave{A}$ l'origine de ce comportement pourrait se trouver la chronologie du développement floral qui aboutit à créer des gradients de développement dans l'épi, entre les épillets et à l'intérieur d'un épillet, entre les différentes fleurs (Kirby, 1974).

La transformation des bourgeons d'épillets en apex d'épillets commence dans le secteur médian de l'épi et se propage de manière acropète et basipète. En revanche, la différenciation florale des épillets, comprenant la réalisation d'un pas floral par thermochrone, est acropète (Malvoisin, 1984a). Elle commence par les épillets les plus avancés. Au moment de la transformation de l'apex de l'épi en épillet terminal, ce qui fixe définitivement la taille de l'épi, les épillets 
médians se trouvent au stade $\mathrm{CO}$, apparition des bourgeons floraux ou $\mathrm{C} 1$, ébauches d'anthères sur les fleurs les plus avancées (Vincent, 1982; Malvoisin, 1984a). D'après Whingwiri et Stern (1982), les fleurs initiées après la formation du bourgeon de l'épillet terminal ne survivent pas à cause de la compétition instaurée entre les différents éléments de l'épi.

Notons aussi que Puckridge (1968) a constaté une vitesse de développement plus faible pour les épillets de la base de l'épi, qui souvent arrêtent leur différenciation au niveau d'une paire de glumes. De la même manière, les fleurs en position distale dans l'épillet ont une vitesse de développement plus faible que celles de la base et dégénèrent avant d'arriver à la fécondation (Langer et Hanif, 1973).

Dans notre essai, en analysant la relation existant entre le nombre de fleurs présentes dans l'épillet à la récolte et le nombre de grains produits par épillet, selon la position de l'épillet et le traitement (NL, Ln, NI), nous avons enregistré une forte corrélation linéaire entre ces deux variables : le nombre de grains par épillet (NGEp) est fonction du nombre total de fleurs de l'épillet (NFEp) (fig 10) :

$N L: N G E p=0,996$ NFE $p-3,12 ; n=5 ; r=0,97$, $L n: N G E p=0,745$ NFEp $-1,63 ; n=5 ; r=0,98$, $N I: N G E p=1,031$ NFE $p-3,62 ; n=5 ; r=0,98$, $L N+L n+I N: N G E p=0,848 N F E p-2,30 ; n=15$; $r=0,96$.

La figure 10 nous montre aussi le comportement particulier par rapport aux facteurs du milieu des épillets situés aux 2 extrémités de l'épi.

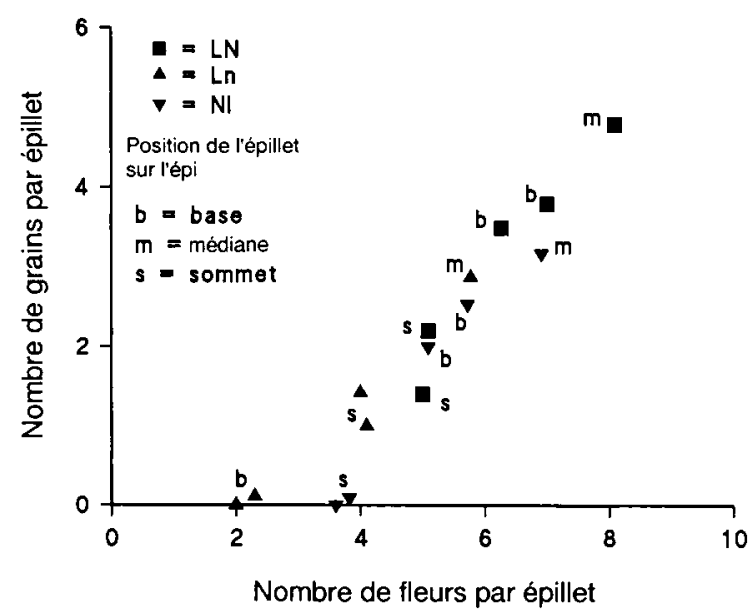

Fig 10. Effet du traitement et de la position de l'épillet dans l'épi sur le nombre de fleurs et de grains par épillet. NL, $n L$, $\mathrm{NI}=$ abréviations explicitées dans l'annexe.
Cependant on peut considérer que chaque épillet possède en moyenne 2-3 fleurs stériles. En supposant que les fleurs stériles soient les plus jeunes de l'épillet, il résulterait qu'une fleur pourrait évoluer vers le grain seulement si elle a atteint un certain seuil de développement.

L'existence d'un stade limite de développement pourrait être déduite aussi à partir des travaux de Langer et Hanif (1973). Sur 9 fleurs initiées dans l'épillet médian, 5 ont arrêté leur développement précocement, peut être avant la méiose; parmi elles, seulement 2 ont commencé la croissance des organes floraux, et sont donc reconnaissables en tant que structures florales à la récolte. L'azote n'a pas eu d'effet sur cet avortement précoce. Les 4 fleurs restantes ont continué leur développement. Elles ont produit 3 grains, même en carence azotée. Ces résultats sont donc très proches des nôtres car ils suggèrent la persistance dans l'épillet à la récolte de 2-3 fleurs stériles, les autres fleurs étant fertiles.

Ainsi le nombre de grains par épillet, et donc par épi, serait déterminé en grande partie par le nombre total de fleurs, par leur vitesse de développement et par leur capacité à atteindre un stade minimal de développement. Plus un organe commence sa différenciation tardivement, plus il aurait besoin d'une vitesse élevée de développement pour arriver à fructifier. L'arrêt précoce du développement floral, aux stades de différenciation des étamines et apparition des lodicules (5-6) (Langer et Hanif, 1973) et plus généralement avant la méiose, suggère aussi une sensibilité accrue de cette phase à différents facteurs du milieu; cela pourrait expliquer l'effet négatif important d'un faible rayonnement à partir de l'apparition de l'avant dernière feuille (fig 10).

Les causes de ces différences et le ou les phénomènes physiologiques responsables de l'arrêt du développement et de la régression des organes restent inconnus, et nos données ne permettent de tirer aucune conclusion sur le déterminisme de ces variations.

La capacité photosynthétique, la translocation et la répartition des assimilats entre les organes n'ont pas encore offert d'explications irréfutables.

L'existence des mécanismes corrélatifs (Whingwiri et Stern, 1982), souvent de nature hormonale et sous contrôle génétique (Trione et Sayavedra-Soto, 1988), dont l'expression est influencée par le milieu, sont les hypothèses les plus plausibles aujourd'hui. 


\section{ANNEXE}

(Récapitulatif des abréviations utilisées dans le texte)

\section{Stades de développement}

A1, At : apparition des ébauches d'entre-nœuds,

A2, Ae, dr : Apparition du bourgeon d'épillet, stade double ride,

b : apparition de la glume,

ET : transformation de l'apex terminal en épillet,

af : apparition de l'avant dernière feuille,

$\mathrm{m}$ : méiose,

ep : épiaison,

$\mathrm{fl}$ : floraison.

\section{Traitements expérimentaux}

LN : lumière naturelle et azote optimal depuis le semis,

$\mathrm{NI}$ : azote optimal et lumière réduite depuis le semis, Ln : lumière naturelle et faible nutrition azotée depuis le semis,

$\mathrm{NLi}$ ou LNi : suppression de l'ombrage ou de la carence azotée à partir du stade de développement «i», $i=d r, b, a f, m, e p, f l$.

\section{D'autres abréviations}

QT: thermochrone,

$S F$ : stade foliaire,

$N G E$ : nombre de grains par épi,

NGEp : nombre de grains par épillet,

NFEp : nombre de fleurs par épillet,

$T R$ : taux de récupération pour la lumière $(T R I)$ et pour l'azote (TRn),

$T P$ : taux de perte pour la lumière $(T P l)$ et pour l'azote $(T P n)$.

\section{RÉFÉRENCES}

Blondon F, Morris $M$ (1985) Actions en phytotron de la vernalisation et d'éclairements forts sur la structure de l'épi de deux variétés de blé tendre d'hiver (Triticum aestivum $\mathrm{L}$ ). agronomie 5, 785-794

Boone MYL, Rickman RW, Whisler FD (1990) Leaf appearance rates of two winter wheat cultivars under high carbon dioxide conditions. Agron $J$ 82, 718-724
Dale JE, Wilson RG (1978) A comparison of leaf and ear development in barley cultivars as affected by nitrogen supply. J Agric Sci (Camb) 90, 503-508

Dale JE, Wilson RG (1979) The effects of photoperiod and mineral nutrient supply on growth and primordia production at the stem apex of barley seedlings. Ann Bot 44, 537-546

Delécolle R, Gurnade JC (1980) Liaison entre le développement et la morphologie du blé tendre d'hiver. Ann Amelior Plant 30, 4, 479-498

Delécolle R, Hay RKM, Guérif $M$, Pluchard $P$, VarletGrancher C (1989) A method of describing the progress of apical development in wheat, based on the time-course of organogenesis. Field Crop Res 21, $147-160$

Evans LT (1987) Short day induction of inflorescence initiation in some winter wheat varieties. Aust $J$ Plant Physiol 14, 277-286

Ficher RA (1985) Number of kernels in wheat crops and the influence of solar radiation and temperature. J Agric Sci (Camb) 105, 447-461

Frank AB, Bauer A (1984) Cultivar, nitrogen, and soil water effects on apex development in spring wheat. Agron J 76, 656-660

Gallagher JN (1979) Field studies on cereal leaf growth. I. Initiation and expansion in relation to temperature and ontogeny. J Exp Bot 30, 625-636

Griffiths EW, Lyndon RF, Bennett MD (1985) The effects of vernalisation on the growth of the wheat shoot apex. Ann Bot 56, 501-511

Hay RKM (1986) Sowing date and the relationships between plant and apex development in winter cereals. Field Crop Res 14, 321-337

Holmes DP (1973) Inflorescence development of semidwarf and standard height wheat cultivars in different photoperiod and nitrogen treatments. Can $J$ Bot 51, 941-956

Haun JR (1973) Visual quantification of wheat development. Agron J65, 116-119

Kirby EJM (1974) Ear development in spring wheat. J Agric Sci (Camb) 82, 437-447

Kirby EJM, Appleyard M, Fellowes G (1982) Effect of sowing date on the temperature response of leaf emergence and leaf size in barley. Plant Cell Environ 5, 477-484

Kirby EJM (1984) Significant stages of ear development in winter wheat. In: Wheat growth and modelling. (W Day and RK Atkin, eds) NATO ASI series, Series A, Life sciences, 86, 7-24

Kirby EJM, Appleyard M, Fellowes G (1985a) Effect of sowing date and variety on main shoot leaf emergence and number of leaves of barley and wheat. agronomie 5, 117-126

Kirby EJM, Appleyard M, Fellowes G (1985b) Leaf emergence and tillering in barley and wheat. agronomie 5, 193-200

Kirby EJM, Siddique KHM, Perry MW, Kaesehagen D, Stern WR (1989) Variation in spikelet initiation and 
ear development of old and modern Australian wheat varieties. Field Crop Sci 20, 113-128

Langer HM, Hanif M (1973) A study of floret development in wheat (Triticum aestivum L). Ann Bot 37 , 743-751

Malvoisin P (1984a) Organogenèse et croissance du maitre brin du blé tendre (Triticum aestivum) du semis à la floraison. I. Relations observées entre la croissance foliaire et la différenciation des ébauches foliaires ou florales. agronomie 4, 557564

Malvoisin $P$ (1984b) Organogenèse et croissance du maître brin du blé tendre (Triticum aestivum) du semis à la floraison. II. Contrôle des relations entre la croissance et la vascularisation de la tige et des feuilles. Essai de modélisation. agronomie 4, 587596

McMaster GS, Morgan JA, Willis WO (1987) Effects of shading on winter wheat yield, spike characteristics, and carbohydrate allocation. Crop Sci 27, 967-973

Miglietta F (1989) Effect of photoperiod and temperature on leaf initiation rates in wheat (Triticum spp). Field Crop Res 21, 121-130

Miglietta F (1991a) Simulation of wheat ontogenesis: I. Appearance of main steam leaves in the field. Clim Res 1, 145-150

Miglietta F (1991b) Simulation of wheat ontogenesis: II. Effect of variety, nitrogen fertilization and water stress on leaf appearance and final leaf number in the field. Clim Res 1, 233-242

Porter RJ, Kirby EJM, Day W, Adam JS, Appleyard Margaret, Ayling Sarah, Baker CK, Beale P, Belford RK, Biscoe PV, Chapman A, Fuller MP, Hampson Janice, Hay RKM, Hough MN, Matthews S, Thompson WJ, Weir AH, Willington Anne VB, Wood DW
(1987) An analysis of morphological development stages in Avalon winter wheat crop with different sowing dates and at ten sites in England and Scotland. J Agric Sci (Camb) 109, 107-121

Puckridge DW (1968) Competition for light and its effect on leaf and spikelet development of wheat plants. Aust J Agric Res 19, 191-201

Rahman MS, Wilson JH (1978) Determination of spikelet number in wheat. III. Effect of varying temperature on ear development. Aust J Agric Res 29, 459-467

Rawson HM, Bagga AK (1979) Influence of temperature between floral initiation and flag leaf emergence on grain number in wheat. Aust $J$ Plant Phy siol 6, 391-400

Siddique KHM, Kirby EJM, Perry MV (1989) Ear stem ratio in old and modern wheat varieties; relationship with improvement in number of grains per ear and yield. Field Crops Res 21, 59-78

Triboï E (1990) Modèle d'élaboration du poids du grain chez le blé tendre (Triticum aestivum em Thell). agronomie 10, 191-200

Trione EJ, Sayavedra-Soto LA (1988) Wheat development enhanced by hormone syndrome. Bot Gaz $149,317-324$

Vincent A, Farcy E, Suty L (1977) Sur la logique du blé. Doc INRA station d'amélioration des plantes, Dijon

Vincent A (1982) Morphogenèse du blé maitre brin. Groupe blé, janvier 1982, 41 p, 25 fig

Whingwiri EE, Stern WR (1982) Floret survival in wheat: significance of the time of floret initiation relative to terminal spikelet formation. J Agric Sci (Camb) 98, 257-268 